\title{
NEW CHARACTERIZATIONS OF APPROXIMATELY GORENSTEIN RINGS
}

\author{
by JOHNNY A. JOHNSON and MONTY B. TAYLOR
}

(Received 26 June, 1991)

In [1], a condition was sought on a commutative Noetherian ring $R$ such that whenever $R$ satisfied the condition and $R$ was cyclically pure in an $R$-algebra $S$, then $R$ was necessarily pure in $S$. Such a condition was found and turned out not only to be a sufficient condition but also a necessary condition. Rings satisfying this condition were called approximately Gorenstein. In this paper, we give new equivalent conditions for a ring to be approximately Gorenstein. Our conditions involve a certain metric topology defined on the lattice of ideals of a commutative Noetherian ring as well as the concept of a principal system. The notion of principal systems was defined in [9] and was suggested by Macaulay's theory of inverse systems [7]. These ideas have proved to be useful in many research probems in commutative algebra.

Throughout this paper all rings are commutative and have a multiplicative identity. In general we adopt the ring terminology of [5] and [8] (in particular, a local ring is Noetherian). Let $R$ be a local ring with maximal ideal $m$. For an ideal $a$ of $R$, we define $Q_{a}$ to be the set of all irreducible $m$-primary ideals of $R$ which contain $a$ (an irreducible ideal is an ideal which cannot be expressed as the intersection of two strictly larger ideals). A metric $d$ (called the $m$-adic metric) can be defined on the lattice of ideals of $R$, $\mathscr{L}(R)$, as follows (where $m^{0}=R$ ):

$$
d(a, b)=0 \text { if } a+m^{n}=b+m^{n} \text { for every natural number } n ;
$$

and otherwise,

$$
d(a, b)=2^{-s(a, b)} \quad \text { where } \quad s(a, b)=\sup \left\{n \mid a+m^{n}=b+m^{n}\right\} .
$$

The $m$-adic metric gives rise to the $m$-adic topological completion of the lattice $\mathscr{L}(R)$ of ideals of $R$ [3] and occurs naturally in various ring theoretic situations (e.g. [2] and [4]).

The following definition of principal system was given by Northcott and Rees in [9]. A proper ideal $a$ of a local ring $R$ with maximal ideal $m$ is a principal system if for every $m$-primary ideal $q$ containing $a$, there exists an irreducible $m$-primary ideal $q^{\prime}$ of $R$ satisfying $a \subseteq q^{\prime} \subseteq q$. We will later use the following result.

LEMMA 1. Let a be an ideal in a local ring $R$ with maximal ideal $m$. A necessary and sufficient condition for a to be a principal system in $R$ is that a is a limit point of $Q_{a}$ in the $m$-adic topology on the lattice of ideals $\mathscr{L}(R)$ of $R$.

Proof. Assume that $a$ is a principal system in $R$ and that $\varepsilon>0$. Choose a positive integer $N$ so that $2^{-N}<\varepsilon$. Then $a+m^{N}$ is an $m$-primary ideal of $R$ containing $a$ since $\operatorname{Rad}\left(a+m^{N}\right) \supseteq \operatorname{Rad}\left(m^{N}\right)=m$. So there exists an irreducible $m$-primary ideal $q^{\prime}$ of $R$ such that $a \subseteq q^{\prime} \subseteq a+m^{N}$. Thus, $a+m^{N}=q^{\prime}+m^{N}$, so it follows that $q^{\prime} \in Q_{a}$ and $d\left(a, q^{\prime}\right)<$ $2^{-N}<\varepsilon$. Thus, $a$ is a limit point of $Q_{a}$ in the $m$-adic topology on $\mathscr{L}(R)$. Conversely, assume that $a$ is a limit point of $Q_{a}$ in the $m$-adic topology on $\mathscr{L}(R)$. Further assume that $q$ is an $m$-primary ideal of $R$ containing $a$. Choose a positive integer $K$ such that $m^{K} \subseteq q$. By hypothesis there exists $q^{\prime} \in Q_{a}$ such that $d\left(a, q^{\prime}\right)<2^{-K}$, and so $a+m^{K}=q^{\prime}+m^{K}$. So $a \subseteq q^{\prime} \subseteq q^{\prime}+m^{K}=a+m^{K} \subseteq q$. Thus, $a$ is a principal system in $R$, which completes the proof. 
Let $R$ be a local ring with maximal ideal $m$. Following [5], we will call $R$ Gorenstein if its injective dimension is finite. Furthermore, we will say that $R$ is approximately Gorenstein [1] if for every positive integer $N$ there is an ideal $q$ of $R$ such that $q \subseteq m^{N}$ and $R / q$ is Gorenstein. We first give our characterizations of approximately Gorenstein local rings. These characterizations use the properties of the $m$-adic topology on the lattice of ideals $\mathscr{L}(R)$ of a local ring $R$ as well as the concept of principal systems discussed above.

THEOREM 2. Let $R$ be a local ring with maximal ideal $m$. Then the following are equivalent:

2.1. $R$ is approximately Gorenstein.

2.2. For every positive integer $k$, there exists an irreducible m-primary ideal $q$ of $R$ such that $q \subseteq m^{k}$.

2.3. $\{0\}$ is a limit point of the set of all irreducible $m$-primary ideals of $R$ in the $m$-adic topology on $\mathscr{L}(R)$.

2.4. $\{0\}$ is a principal system in $R$.

Proof. The equivalence of 2.1 and 2.2 was shown in [1, (2.2) Corollary]. In addition, by Lemma 1 , we have that 2.3 and 2.4 are equivalent. We now show that 2.2 implies 2.4 . Suppose 2.2 holds. Suppose further that $q$ is an $m$-primary ideal of $R$. Pick a positive integer $k$ such that $m^{k} \subseteq q$. Using 2.2, there exists an irreducible $m$-primary ideal $q^{\prime}$ of $R$ such that $q^{\prime} \subseteq m^{k}$. Hence, $q^{\prime} \subseteq q$ and thus $\{0\}$ is a principal system in $R$. Therefore, 2.2 implies 2.4. To complete the proof, we show that 2.2 follows from 2.3. Suppose 2.3 holds. Suppose further that $k$ is a positive integer. Then by 2.3 , there exists an irreducible $m$-primary ideal $q$ of $R$ such that $d(\{0\}, q)<2^{-k}$. It follows that $q \subseteq q+m^{k}=\{0\}+m^{k}=$ $m^{k}$. Hence, 2.3 implies 2.2, which completes the proof of the theorem.

For a local ring $R$, we let $R^{*}$ denote the ring completion of $R$ in its natural topology [8]. Since the zero ideal of $R$ is a principal system in $R$ if and only if it is a principal system in $R^{*}$, one immediately obtains the following corollary.

COROLlary 3. Let $R$ be a local ring. Then $R$ is approximately Gorenstein if and only if $R^{*}$ is approximately Gorenstein.

A Noetherian ring $R$ is said to be approximately Gorenstein if the local ring $R_{m}$ is approximately Gorenstein for every maximal ideal $m$ of $R$ [1]. We now proceed to globalize our previous results and give characterizations of arbitrary Noetherian approximately Gorenstein rings. These characterizations are also in terms of principal systems and certain topologies on particular ideal lattices.

THeORem 4. Let $R$ be a Noetherian ring. Then the following are equivalent:

4.1. $R$ is approximately Gorenstein.

4.2. For every maximal ideal $m$ of $R,\{0\}$ is a principal system in $R_{m}$.

4.3. For every maximal ideal $m$ of $R,\{0\}$ is a limit point of the set of all irreducible $m R_{m}$-primary ideals of $R_{m}$ in the $m R_{m}$-adic topology on $\mathscr{L}\left(R_{m}\right)$.

Proof. This is easily seen to follow from Theorem 2 and the definition of a Noetherian approximately Gorenstein ring. 
Let $R$ be a Noetherian ring. Then $R$ is a Zariski ring with respect to the Jacobson radical of $R$. We let $R^{*}$ denote the completion of $R$ in the Jacobson radical topology. The following theorem extends the local case result of Corollary 3 .

THEOREM 5. Let $R$ be a Noetherian ring. Then $R$ is approximately Gorenstein if and only if $R^{*}$ is approximately Gorenstein.

Proof. Let $\operatorname{Max}(R)$ denote the collection of all maximal ideals of $R$. Let $\operatorname{Max}\left(R^{*}\right)$ denote the collection of all maximal ideals of $R^{*}$. By $[6, \S 24 \mathrm{~A}]$, note that $\operatorname{Max}\left(R^{*}\right)=$ $\left\{m R^{*} \mid m \in \operatorname{Max}(R)\right\}$. From the definition of approximately Gorenstein given above and Corollary 3 , we have that $R$ is approximately Gorenstein if and only if for each $m \in \operatorname{Max}(R),\left(R_{m}\right)^{*}$ is approximately Gorenstein. Similarly, we have that $R^{*}$ is approximately Gorenstein if and only if for all $m \in \operatorname{Max}(R),\left(R_{m R^{*}}^{*}\right)^{*}$ is approximately Gorenstein. Furthermore, from $[6, \S 24 . \mathrm{D}]$, it follows that for each $m \in \operatorname{Max}(R),\left(R_{m}\right)^{*}$ is isomorphic to $\left(R_{m R^{*}}^{*}\right)^{*}$. By combining these observations, we obtain the desired result.

\section{REFERENCES}

1. M. Hochster, Cyclic purity in excellent Noetherian rings, Trans. Amer. Math. Soc. 231 (1977), 463-487.

2. E. W. Johnson, Modules: duals and principally generated fake duals, Algebra Universalis 24 (1987), 111-119.

3. E. W. Johnson and J. A. Johnson, Lattice modules over semi-local Noether lattices, Fund. Math. 68 (1970), 187-201.

4. J. A. Johnson, A note on semilocal rings, Proc. Amer. Math. Soc. 55 (1976), 469-470.

5. I. Kaplansky, Commutative rings (Allyn and Bacon, Boston, 1970).

6. H. Matsumura, Commutative algebra, second edition, (Benjamin/Cummings, Reading, Massachusetts, 1980). 1916).

7. F. S. Macaulay, The algebraic theory of modular systems (Cambridge Tracts in Math. 19,

8. M. Nagata, Local rings, Interscience Tracts in Pure and Appl. Math., no. 13 (Interscience, New York, 1962). 119-127.

9. D. G. Northcott and D. Rees, Principal systems, Quart. J. Math. Oxford (2), 8 (1957),

Department of Mathematics

UNIVERSITY OF HOUSTON

Houston, TX 77204-3476
Department of Mathematics and Computer Science UNIVERSITY OF TEXAS-PAN AMERICAN

EDINBURG, TX 78539-2999 